\title{
Six-months pain relief and functional recovery after intra-articular injections with hyaluronic acid (mw 500-730 KDa) in trapeziometacarpal osteoarthritis
}

\author{
Antonio Frizziero ${ }^{1}$ \\ Nicola Maffulli ${ }^{2}$ \\ Stefano Masiero' \\ Luigi Frizziero ${ }^{3}$
}

1 Department of Physical and Rehabilitation Medicine, University of Padova, Italy

2 Queen Mary University of London Centre for Sports and Exercise Medicine Mile End Hospital Mann Ward, London, UK; Head of Department of Physical and Rehabilitation Medicine, University of Salerno, Azienda Ospedaliera San Giovanni di Dio e Ruggi d'Aragona, Salerno, Italy

3 Department of Rheumatology, Villa Toniolo Hospital, Bologna, Italy

Corresponding author:

Antonio Frizziero

Department of Physical and Rehabilitation Medicine, University of Padova

Via Giustiniani, 2

35128 Padova, Italy

E-mail: antonio.frizziero@gmail.com

Summary

Background: this retrospective open label study evaluates the efficacy and tolerability of intra-articular injections of Hyaluronic Acid (HA) (MW 500-730 KDa - Hyalgan $\AA$ ) for the treatment of pain and disability of trapeziometacarpal joint osteoarthritis (TMCJ OA).

Methods: fifty eight patients, 50 females (86\%) and 8 male $(14 \%)$, aged between $40-75$ years, suffering from TMC $J$ OA according to Kellgren-Laurence grades 2-3 on standard plain radiography, were included. Patients with known inflammatory arthritis, previous thumb trauma and intra-articular (i.a.) injections with corticosteroids were excluded. Primary endpoints were: pain (VAS), NSAID intake, radial and palmar ab-/adduction, pinch strength. All patients received an i.a. injection of $0.8 \mathrm{~mL}$ of $\mathrm{HA}$ (MW 500-730 KDa) once weekly for three weeks. Control examinations were carried out at 1,3 , and 6 months. Results: intra-articular HA injections have significantly reduced spontaneous and provoked pain and improved hand mobility. At 1,3, and 6 months from baseline, the spontaneous and provoked pain revealed a statistically significant improvement $(p<0,0001)$. NSAID's intake evidenced a statistically significant reduction against baseline $(p<0.017)$. The adverse events $(21 \%)$ were related to local symptoms such as pain during or following $\mathrm{HA}$ administration.

Conclusions: this study shows that i.a. HA injections for TMCJ OA can induce a significant improvement of function associated to stiffness decrease and pain relief.

KEY WORDS: trapeziometacarpal joint, osteoarthritis, hyaluronic acid.

\section{Introduction}

Hand OA, common in Western populations, is a multifactorial condition in which age, sex and genetic factors play an important role ${ }^{1}$. The prevalence of TMCJ $\mathrm{OA}$ is 8 to $12 \%$ in the general population, and as high as $33 \%$ in post-menopausal women ${ }^{2}$. It is associated with pain and site-specific disability, including interference with grip and fine precision pinch, along with the presence and progression of OA at other sites, particularly the $\mathrm{knee}^{3}$. It is often non responsive to standard medical treatment, and many patients need surgery 4 . TMCJ OA occurs most frequently in the non-dominant hand: activity may protect against hand $O A$. Hand OA is highly associated with the presence and progression of OA in the knee but not in the hip ${ }^{3,5,6}$.

Current therapeutic options primarily aim at reducing pain and improving joint function using symptomatic agents. Within the possible therapeutic approaches, intra-articular (i.a.) injection of Hyaluronic Acid (HA) (MW 500-730 KDa - Hyalgan ${ }^{\circledR}$ ) has proven to be safe and effective for patients with hand $O A^{6}$, and may provide an effective and safe alternative to corticosteroids (CS) in patients at high risk of side effects caused by CS treatment. On articular cartilage, HA acts as a structural element by producing space and re-organizing the tissue. In the synovial fluid, HA unique viscoelastic properties provide joint lubrication and shock absorption 7 . Some studies have suggested that i.a. injections of HA may reduce pain and improve function in patients with $O A^{6,8}$, but other authors reported that the effect of i.a. HA was no better than those of i.a. CS or injection of normosaline ${ }^{9,10}$. However, a systematic review of available data pro- 
vide further evidence that HA injections may be an effective low-risk treatment for TMCJ OA unresponsive to standard medical therapy ${ }^{4,11-13}$.

This retrospective open label study evaluated the results of i.a. HA (MW 500-730 KDa) injections, in terms of pain relief and hand function improvement, in patients suffering from TMCJ OA in whom previous standard medical therapy had been unsuccessful.

\section{Patients and methods}

This open-label retrospective study was approved by the Ethics Committee for Medical Research of Local Health Authority of Bologna and meets the ethical standards of this journal ${ }^{14}$.

All patients provided written informed consent and were free to withdraw from the treatment at any time. In the period within January 2000 and December 2002 a total of 58 patients, 50 females $(86,2 \%)$ and 8 males $(13,8 \%)$, within 40 and 75 years old with a mean age of 57,0 years $( \pm 8.4)$ and a mean BMI of $24.5( \pm 2.1)$, were screened and included in the study (Tab. 1). Forty-six patients $(79,3 \%)$ reported systemic OA. All patients suffered of pain and tenderness of TMC joint, and 50 of these 58 patients $(86,2 \%)$ suffered from a bilateral condition with a total of 108 joints treated (Tab. 2). X-rays examination showed radiographic evidence of joint space narrowing, scle-

Table 1. Demographic characteristics at baseline.

\begin{tabular}{lrrr}
\hline & Mean & Median & St. dev \\
\hline Age & 57.09 & 56.00 & 8.436 \\
BMI & 24.58 & 24.67 & 2.099 \\
Weight (Kg) & 66.84 & 66.00 & 9.047 \\
Height & 164.64 & 164.00 & 6.595 \\
\hline
\end{tabular}

Table 2. Patient's OA condition at baseline.

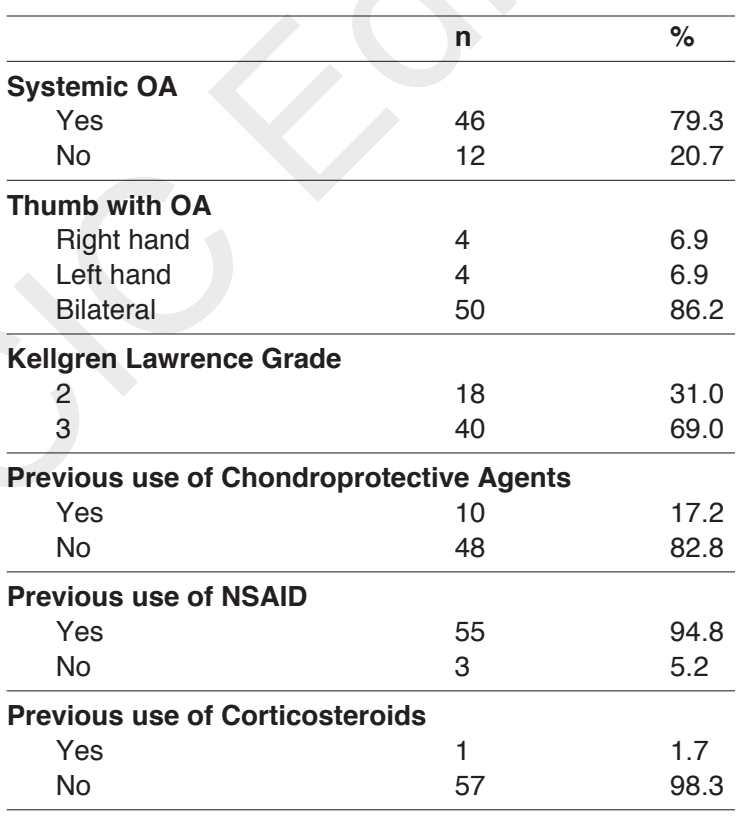

rosis and osteophyte presence according to KellgrenLawrence score grade 2 (31,0\%of patients) or 3 $(69,0 \% \text { of patients })^{15}$. The follow-up period of at least 6 months has been established on the base of the Schumacher et al. previous experience in which it has been shown that HA MW 500-730 KDa has a long lasting pain relief up to six months ${ }^{16}$. All patients fulfilled the diagnostic criteria of OA according to EULAR recommendations ${ }^{17}$.

Were excluded from this study patients with arthritic or metabolic pathologies and with serious previous trauma, patients with a follow-up period of less than 6 months, and patients with clinically relevant deformity. Fifty five of 58 patients included $(94,8 \%)$ responded insufficiently to prior treatments with Non-Steroidal Anti-Inflammatory Drugs (NSAIDs) taken regularly, while three patients had never assumed any drug to treat the TMCJ pain. To all patients the same drugs was given during the study as rescue drug only for TMCJ pain.

All patients underwent one cycle of 3 weekly i.a. injections of $0.8 \mathrm{~mL}(10 \mathrm{mg} / \mathrm{mL}$ ) of $\mathrm{HA}$ (MW 500-730 $\mathrm{KDa}$ ) using a dorsolateral approach after palpating the TMC joint space. The volume of $0,8 \mathrm{~mL}$ of HA induced the least pain when injected ${ }^{8}$. The TMC joints were injected with a 22-gauge needle after skin cleansing with $10 \%$ povidone iodine and then spraying with ethyl chloride.

The efficacy of the i.a. injections of HA was assessed evaluating pain during the day at rest and on voluntary or passive movements of flexion, extension, abduction and rotation using a $10-\mathrm{cm}$ Visual Analog Scale (VAS), and through standard goniometer. Lateral pinch strength was measured using a Jamar Model 1 hand dynamometer (TEC, Clifton, NJ). The morning stiffness lasting and NSAIDs consumption were assessed through direct questionnaire (pills/days/month). Other symptoms recorded were swelling and crepitation under palpation. Pain related to the i.a. injection procedure was assessed using VAS. Also, patient's and investigator's global assessment were collected. To assess safety, adverse events occurring immediately after the treatment or during the follow up period were recorded throughout the study.

At baseline and at each subsequent observation times, at 1, 3 and 6 months after the treatment, all the efficacy and safety parameters were assessed by the same blinded investigator.

Statistical analysis: to demonstrate the efficacy of i.a. HA injections in TMCJ OA treatment, changes from baseline for each study outcomes were calculated at each study point at 1, 3, and 6 months. Mean, median, standard deviation, and range are reported for continuous variables, count and proportions are reported for discrete variables.

Given the nature of measured variables and their distribution at baseline, to investigate the outcome measures parametric and non parametric tests were applied (Tab. 3). All comparisons were conducted against baseline with significance set at a level of 0.05 . 


\section{A. Frizziero et al.}

Table 3. Statistical test applied in data analysis.

\begin{tabular}{ll}
\hline Variable & Test \\
\hline Pain (spontaneous,- at rest, on use, during day and night) & Wilcoxon test \\
Palmar Adduction and Radial Abduction & Analysis of variance for repeated measures \\
Pinch strength & Analysis of variance for repeated measures \\
Morning stiffness & Analysis of variance for repeated measures \\
Swelling & McNeemar test \\
NSAIDs' intake & Wilcoxon test \\
Patient's and physician's global assessments & Wilcoxon test \\
\hline
\end{tabular}

When changes from baseline were assessed by the Wilcoxon test the a level was adjusted for 3 comparisons to an $\mathrm{a}=0.017$.

\section{Results}

Prior to HA administration, pain VAS score for voluntary activity was $9.3 \mathrm{~cm}( \pm 0.6)$, for passive activity was $9.9 \mathrm{~cm}( \pm 0.3)$, and $8.2 \mathrm{~cm}( \pm 0.7)$ at night. A statistically significant improvement of the spontaneous and pain during activity at all follow up visits (Wilcoxon test, $p<0.001$ ) was observed.

At month 1, the mean value of pain for voluntary activity dropped to $5.9 \mathrm{~cm}( \pm 2.0)$, then increased to $6.5 \mathrm{~cm}( \pm$ $2.3)$ and to $7.7 \mathrm{~cm}( \pm 1.8)$ at 3 and 6 months respectively. Moreover, provoked pain during passive activity mean value dropped to $7.0 \mathrm{~cm}( \pm 1.9)$ one month after the first treatment, then increased to $7.4 \mathrm{~cm}( \pm 2.0)$ and to $8.3 \mathrm{~cm}( \pm 1.7)$ at 3 and 6 months respectively (Fig. 1). At month 1 , the mean value of pain during the night time dropped to $4.7 \mathrm{~cm}( \pm 2.2)$, then increased to 5.3 $\mathrm{cm}( \pm 2.3)$ and to $6.5 \mathrm{~cm}( \pm 1.9)$, at 3 and 6 months, respectively (Fig. 2).

At the first evaluation point, at month 1, morning stiffness were reported to have decreased from an average of $15.0( \pm 5.2)$ to $9.4( \pm 5.3)$ minutes; radial abduction increased from a baseline value of $19.8( \pm 8.6)$ up to $22.5( \pm 8,1)$ degrees, and palmar adduction improved from $29.5( \pm 9.3)$ to $32.0( \pm 8.9)$ degrees. Pinch strength test did not show significant improvements. At month 3, morning stiffness was reduced, lasting an average of $8.9( \pm 4.1)$ minutes; radial and palmar ablabduction maintained stable values and the percentage of patients in whom the swelling completely disappeared increased to 55\% (Fig. 3).

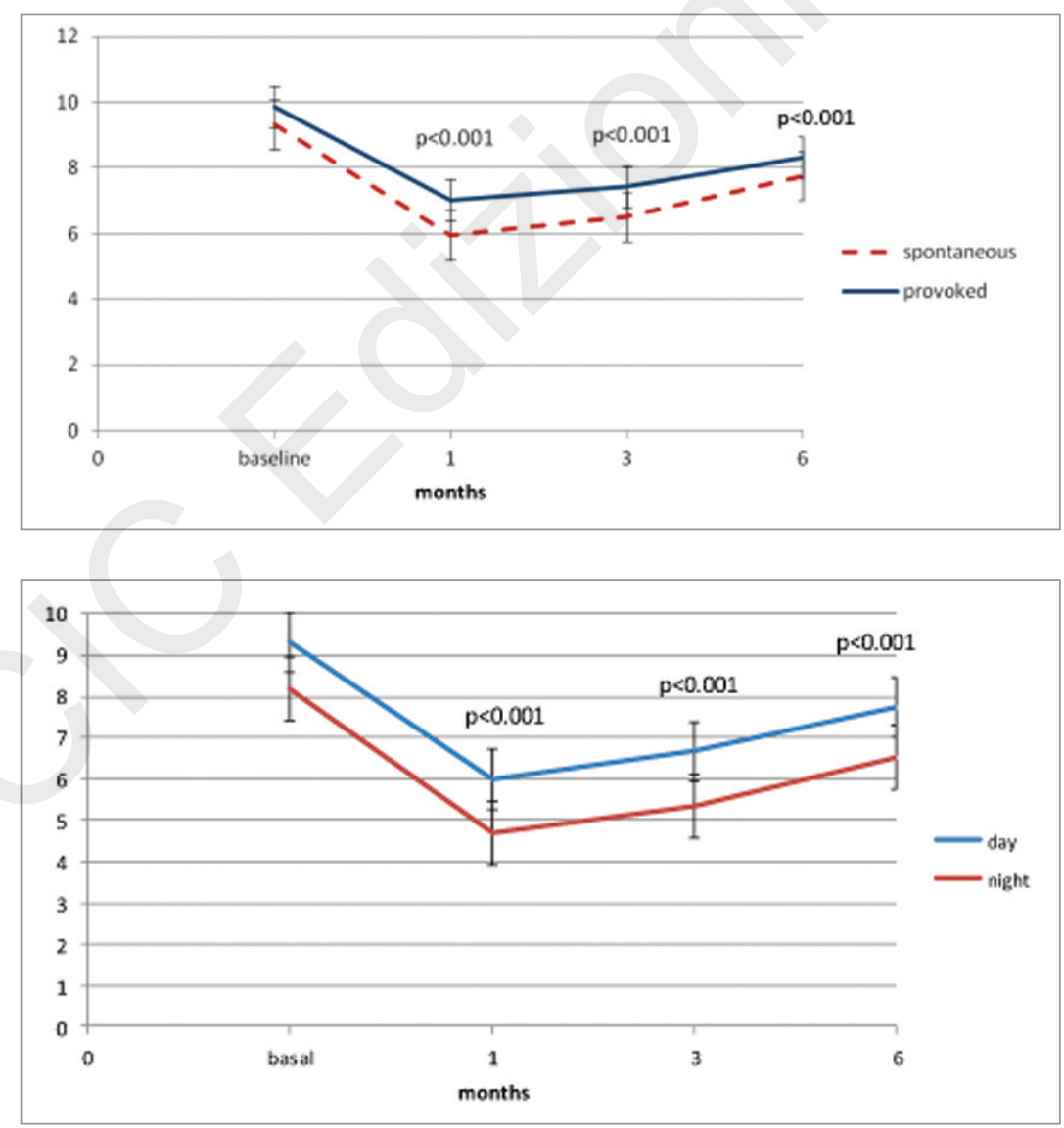

Figure 1. Mean values of pain on a 0-10 Visual Analogue Scale (VAS) perceived during voluntary and passive activity.

Figure 2. Mean values of pain on a 0-10 Visual Analogue Scale (VAS) perceived during day and night. 
At the final visit, morning stiffness lasted an average of $11.3( \pm 4.6)$ minutes; the movements of radial and palmar ab-/adduction slightly decreased to 21.7 ( \pm $8.6)$ and $31.0( \pm 8.9)$ degrees respectively. Fifteen adverse events occurred in 12 patients during the treatment period: 3 patients complained and pain during the injection, 7 patients reported pain after treatment, and 2 patients experienced flushing associated to malaise or tachycardia. All events lasted one day and were of moderate intensity with the exception of a severe pain perceived by one patient after injection. The investigator considered the relationship with the study drug definite in 8 events $(53.5 \%)$, probable in 6 events $(40 \%)$, and possible in one $(7 \%)$.

Thirteen events had completely recovered at the end of the study, while two events were improving or stable at six months from the injection.

Analysis of the variance for radial and palmar ab-/adduction, and morning stiffness evidenced a statistically significant improvement against baseline at all subsequent visits $(p<0.001)$. Furthermore, the Wilcoxon test performed to compare both the days of NSAIDs assumption at baseline against each study point (Fig. 4 ), and the patient's and investigator's global assessments evidenced a statistically significant difference at all evaluation time point: $p<0.017$ and $p<0.001$ respectively.

The McNeemar test, performed to evaluate the percentage of patients changing swelling conditions, showed a statistically significant improvement against baseline at all subsequent visits $(p<0.001)$ (Fig. 5). Pain of moderate intensity during i.a. injection occurred in 12 patients, and in 4 patients lasted 10-30'.

\section{Discussion}

The biological rationale for the use of i.a. HA in osteoarthritis has been widely studied in large joints as a symptom-modifying treatment for its potential to restore synovial fluid viscoelastic properties, and it may also have modifying effects on diseased joints ${ }^{17-19}$. In experimental models of OA, HA treatment showed positive long lasting effects, possibly mediated by the reduction of inflammatory mediators and effects on molecular factors involved in disease progression ${ }^{20}$. HA functions depend on its molecular mass: a molecular weight between $500-730 \mathrm{KDa}$ is associated with anti-inflammatory effects ${ }^{7}$.

Although some authors showed a lack of efficacy of HA injections, other studies confirm its efficacy and safety for the treatment of $O A$, especially comparing the efficacy and safety of i.a. injections of HA versus $\mathrm{CS}^{6,21}$.

Few studies evaluate the efficacy and tolerability of i.a. HA administration in TMCJ8,16. Intra-articular injection of HA is effective in TMCJ OA treatment; however some conflicting results have been reported 4,11,12,22,23. HA injections may provide an effective alternative to steroid injections in TMCJ OA patients ${ }^{24}$. In a prospective randomized trial comparing corticosteroid and HA, Stahl et al. showed equivalent pain relief at rest and with activity for up to 6 months ${ }^{22}$.
In another randomized, prospective, active-controlled, blinded study, 56 patients with symptomatic mild to moderate $\mathrm{OA}$ of the first CMCJ were treated with HA or Triamcinolone over 56 weeks. Both treatments produced similar effects: Triamcinolone induced faster onset of action, whereas HA was more durable ${ }^{6}$.

In a prospective series of 42 patients treated with sodium hyaluronate for symptomatic TMCJ OA, Roux et al. found that VAS pain grades decreased markedly for those patients who received 2 and 3 injections ${ }^{23}$.

A more recent study suggests that saline, steroid and HA injections are effective for symptomatic improvement in basal joint arthritis. HA injection into symptomatic TMCJ OA is a reasonable therapy and can be employed as a final nonsurgical measure ${ }^{11}$.

HA injections in TMCJ OA are well tolerated; adverse events mainly consisted of pain during or following HA i.a. administration; none required active treatments. The reason of pain may be para-articular injection or periosteal irritation and the pain related to the injection procedure can be reduced using fluoroscopic guidance or US-guided procedures ${ }^{8}$. AIthough recent studies show that the accuracy of i.a. injections of TMCJ can be enhanced under ultrasound guidance, in expert hands also blind injections can provide high accuracy, using anatomic landmarks to guide needle placement ${ }^{4}$. Significant improvements in pain and measures of function were evaluated in another prospective, open-label study of a single ultrasound-guided injection of $\mathrm{HA}$ into the first CMCJ in 18 patients with symptomatic OA (K-L grade $2-3)^{8}$.

The results of our study provide further evidence that $\mathrm{HA}$ injection may be an effective low-risk treatment for TMCJ OA unresponsive to standard medical therapy. Results comparable with our experience are reported by a recent work in which i.a. injections of $\mathrm{HA}$ ultrasound-guided are effective in reducing synovial hyperemia significantly correlated with reduced pain only for 6 months, suggesting the possibility to repeat periodic injections ${ }^{25}$.

Schumacher et al. open-label baseline-controlled study of five once-weekly injections of HA (MW 500$730 \mathrm{KDa}$ ) into the first carpal-metacarpal joint (CMCJ) in 16 patients with radiographically verified symptomatic OA, provides preliminary evidence that, 6 months since the beginning of treatment, the mean pain score at rest and on use decreased $46 \%$ and $27 \%$ from baseline respectively. Pinch strength did not significantly improve in any of the patients ${ }^{16}$. This initial report was not blinded and had not control group; a small decrease in pain was noted but no statistical values were reported.

Recently, Salini et al. ${ }^{18}$ showed that pain at rest and during activity decreased from 1.8 to $0.5(p<0,001)$ and from 8.05 to 4.15 ( $p<0,001)$, respectively. In other studies once weekly injection for 3 weeks with moderate TMCJ OA (K-L 2-3 grades) showed significant improvements in disabilities of the hand score and pain at 26 weeks, but key and opposition pinch showed minimal differences compared with baseline ${ }^{4}$. 


\section{A. Frizziero et al.}

\section{Conclusion}

The data presented in this retrospective open-label study suggest that HA (MW 500-730 KDa) i.a. injections can be a valid therapeutic option inducing prolonged and sustained clinical remission of pain with improvement of mobility and increase quality of life in patients suffering from TMCJ OA when conventional therapy with NSAIDs were ineffective or contraindicated, before surgery. Given the current knowledge of the precautions associated with chronic use of oral medications, HA might be considered a first-line therapeutic option when simple analgesic and nonpharmacologic interventions have failed. Our study con-
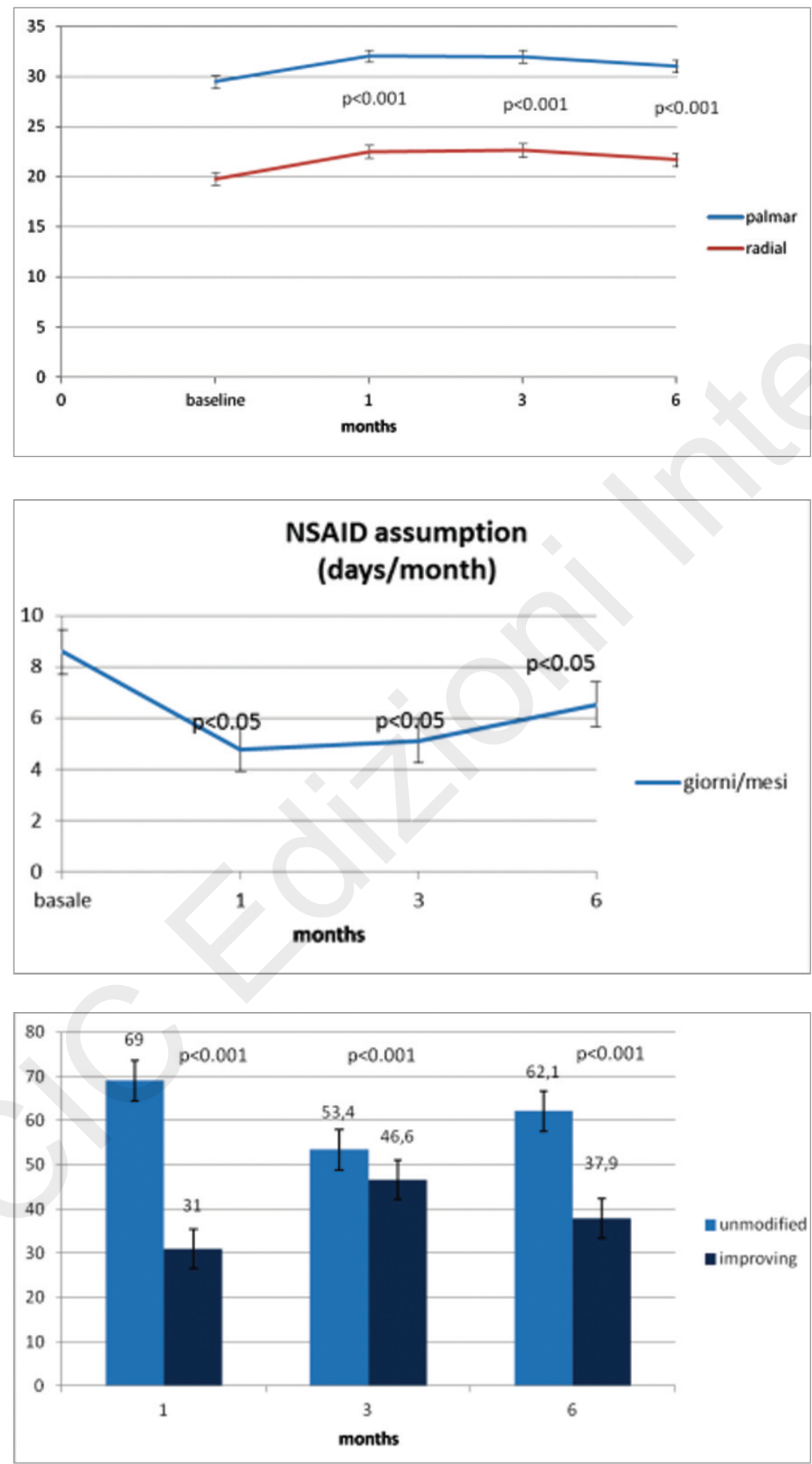

Figure 3. The mean radial abduction and palmar adduction of the target thumb.

Figure 4. Statistically significant reduction of Mean NSAID consumption rate at baseline and at each study point.

Figure 5. Percentage of patients changing swelling conditions from baseline at each study point. 
firms, also, that i.a. injections of HA into TMCJ are performed easily by and expert operator and are a tolerable but it isn't a painless procedure.

We recognize the limits of the study that unfortunately do not include an intra-articular control arm. It could be argued that randomized controlled trials and longer follow-up periods are required to determine whether HA $500-730 \mathrm{KDa}$ is an effective agent in controlling local pain and inflammation of TMCJ OA and in inducing a long term remission.

\section{References}

1. Zhang W, Doherty M, Leeb BF, et al. EULAR evidence based recommendations for the management of hand osteoarthritis: Report of a task force of the EULAR Standing Committee for international clinical studies including therapeutics (ESCISIT). Ann Rheum Dis. 2007;66:377-388.

2. Haara MM, Heliövaara M, Kröger $\mathrm{H}$, et al. Osteoarthritis in the Carpometacarpal joint of the thumb. J Bone Joint Surg. 2004:1452-1457.

3. Jonsson H, Manolescu I, Stefansson E, et al. The inheritance of hand osteoarthritis in Iceland. Arthritis Rheum. 2003; 48:391-395.

4. Mandl LA, Hotchkiss RN, Adler RS, et al. Injectable hyaluronan for the treatment of Carpometacarpal osteoarthritis: an open label pilot trial. Curr Med Res Opin. 2009;25:21032108.

5. Cushnaghan J, Dieppe P. Study of 500 patients with limb joint osteoarthritis: I. Analysis by age, sex and distribution of symptomatic joint sites. Ann Rheum Dis. 1991;50:8-13.

6. Fuchs $\mathrm{S}$, Mönikes R, Wohlmeiner A, et al. Intra-articular hyaluronic acid compared with corticoid injections for the treatment of rhizarthrosis. Osteoarthritis Cartilage. 2006;14: 82-88.

7. Serban MA, Yang G, Prestwich GD. Synthesis, characterization and chondroprotective properties of a hyaluronan thioethyl ether derivative. Biomaterials. 2008;19:1388-1399.

8. Karalezli N, Ogun TC, Kartal S, Sacracgil SN, Yel M, Tuncay I. The pain associated with intraarticular hyaluronic acid injections for trapeziometacarpal osteoarthritis. Clin Rheumatol. 2007;26:569-571.

9. Jorgensen A, Stengaard-Pedersen K, Simonsen O, et al. Intra-articular hyaluronan is without clinical effect in knee osteoarthritis: a multicenter, randomized, placebo-controlled, double-blind study of 337 patients for 1 years. Ann Rheum Dis. 2010;69:1097-1102.

10. DeGroot III H, Uzunishvili S, Weir R, Lomari A, Gomes B. Intraarticular injection of Hyaluronic acid is not superior to saline solution injection ankle arthritis. A randomized, double-blind, placebo-controlled study. J Bone Surg Am. 2012;94:2-8.

11. Heyworth BE, Lee JH, Kim PD, Lipton CB, Strauch RJ, Rosenwasser MP. Hylan versus corticosteroid versus placebo for treatment of basal joint arthritis: a prospective, randomized, double-blind clinical trial. J Hand Surg. 2008;33A:40-48.

12. Hochberg MC, Altman RD, April KT, et al. American college of rheumatology 2012, recommendations for the use of nonpharmacologic and pharmacologic therapies in osteoarthritis of the hand, hip and knee. Arthritis Care \& Research. 2012;64:465-474.

13. Pasquali Ronchetti I, Guerra D, Taparelli F, et al. Morphological analysis of knee synovial membrane biopsies from a randomized controlled clinical study comparing the effects of sodium hyaluronate (Hyalgan $\left.{ }^{\circledR}\right)$ and methylprednisolone acetate (Depomedrol®) in osteoarthritis. Rheumatology. 2001; 40:158-169.

14. Padulo J, Oliva F, Frizziero A, Maffulli N. Muscles, Ligaments and Tendons Journal. Basic principles and recommendations in clinical and field science research. MLTJ. 2013;4: 250-252.

15. Kellgren JH, Lawrence JS. Radiological assessment of osteoarthritis. Ann Rheum Dis 1957;16:494-502.

16. Schumacher HR, Meador R, Sieck M, Mohammed Y. Pilot investigation of hyaluronate injections for first metacarpal-carpal (MC-C) osteoarthritis. JCR. 2004;10:59-62.

17. Zhang W, Doherty M, Leeb BF, et al. EULAR evidence-based recommendations for the diagnosis of hand osteoarthritis: report of a task force of ESCISIT. Ann Rheum Dis. 2009;68:8-17.

18. Salini V, De Amicis D, Abate M, et al. Ultrasound-guided hyaluronic acid injection in carpometacarpal osteoarthritis: short term results. Int J Immunopathol Pharmacol. 2009; 22:455-460.

19. Wolf JM. Injection for trapeziometacarpal Osteoarthritis. JHS. 2010;35A:1007-1009.

20. Waddel DD, Kolomytkin OV, Dunn S, et al. Hyaluronan suppresses IL-1 $\beta$-induced metalloproteinase activity from synovial tissue. Clin Orthop Relat Res. 2007;465:241-248.

21. Foti $\mathrm{C}$, Cisari $\mathrm{C}$, Carda $\mathrm{S}$, et al. A prospective observational study of the clinical efficacy and safety of intra-articular sodium hyaluronate in synovial joints with osteoarthritis. Eur J Phys Rehabil Med. 2011;47(3):407-415.

22. Stahl S, Karsh-Zafrir I, Ratzon N, Rosenberg N. Comparison of intraarticular injection of depot corticosteroid and hyaluronic acid for treatment of degenerative trapeziometacarpal joints. $J$ Clin Rheumatol. 2005;11:299-302.

23. Roux C, Fontas E, Breuil V, Brocq O, Albert C, Euller-Ziegler L. Injection of intra-articular sodium hyaluronidate (Sinovial) into the carpometacarpal joint of the thumb (CMC1) in osteoarthritis. A prospective evaluation of efficacy. Joint Bone Spine. 2007;74:368-372.

24. Bahadir C, Onal B, Dayan VY, Gurer N. Comparison of therapeutic effects of sodium hyaluronate and corticosteroids injections on trapeziometacarpal joint osteoarthritis. Clin Rheumatol. 2009;28:529-533.

25. Klauser AS, Faschingbauer R, Kupferthaler K, et al. Sonographic criteria for therapy follow-up in the course of ultrasound-guided intra-articular injections of hyaluronic acid in hand osteoarthritis. European Journal of Radiology. 2012;81: 1607-1611. 